\title{
MUJERES EN LOS GOBIERNOS LOCALES (1979-2015): \\ UNA APROXIMACIÓN A LA REALIDAD EN LA PROVINCIA DE ALICANTE
}

\author{
María Quiles Bailén \\ mquiles@goumh.umh.es \\ Universidad Miguel Hernández de Elche - España
}

\author{
Anastasia Téllez Infantes \\ atellez@umh.es \\ Universidad Miguel Hernández de Elche - España
}

Recibido: 16-02-2016

Aceptado: 04-06-2016

\section{Resumen}

Este artículo tiene por objeto el acercamiento a la realidad de la situación de la mujer en la cúspide política local de la provincia de Alicante desde 1979 hasta 2015, periodo en el que han transcurrido más de treinta y cinco años de democracia en España y en el que se han llevado a cabo un total de diez convocatorias electorales. Hemos realizado una descripción y análisis de la evolución de la presencia de mujeres en los gobiernos locales de la provincia a lo largo de más de tres décadas, comparando las pautas de esta transformación con las acaecidas en los gobiernos autonómicos y nacionales para poder reflexionar sobre cuál es nuestra situación con respecto a las medias nacional y autonómica en cuanto a presencia femenina al cargo de las alcaldías.

Palabras Clave: Mujer, poder político, género, gobierno local.

\begin{abstract}
This article aims to approach to the reality of the female gender in the local political apex of the province of Alicante since 1979 until 2015. In this period, it has passed more than thirty-five years of democracy in Spain in which there has been carried out ten elections in total. We have made a description and analysis of the evolution of the presence of women in local governments in the province for more than three decades, comparing patterns of this transformation with the prevailing in regional and national governments to be able to reflect about our situation with regard to national and regional averages in terms of presence of women in charge of municipalities.
\end{abstract}

Keywords: Woman, political power, gender, local government. 


\section{Introducción}

La gran heterogeneidad de municipios y las diferencias geográficas, culturales, sociales, estructurales e institucionales que éstos presentan ha comportado que el estudio completo de los entes locales sea un asunto complejo y difícil de tratar. Pese a que la crisis económica y los casos de corrupción política están provocando un cuestionamiento de los valores públicos, entendemos que las potencialidades del plano local como objeto de estudio son enormes (Ruiloba, 2013: 94).

$\mathrm{Y}$ es que resulta obvio que las competencias municipales son muchas y variadas, y por tanto, la evolución histórica de las mismas nos permite ver cómo, cada vez más, los consistorios son instituciones básicas para la mejora de la calidad de vida de la ciudadanía. Los nuevos retos sociales, dentro del mundo globalizado en el que vivimos, son resueltos, en la práctica, por nuestros y nuestras representantes locales, por tanto, el estudio de sus características sociológicas y de su acción de gobierno es fundamental para observar la evolución del sistema democrático español y la capacidad de poder resolver los problemas que la nueva sociedad reclama. En este sentido, los Ayuntamientos han cumplido en los últimos años un papel primordial en la corrección de desequilibrios y carencias sociales, en la mayoría de los casos sin un título competencial que respaldase dichas actuaciones y sin una financiación suficiente para sostener una serie de prestaciones y servicios que inciden en el bienestar de la vecindad. Ello ha provocado, entre otras consecuencias, un importante endeudamiento de las corporaciones locales, en el cual a su vez ha estado el origen de muchas de las situaciones de corrupción que, han sacudido recientemente la vida municipal española (Salazar, 2010: 192).

Con estas premisas no queremos plantear que la dinámica política municipal sea diametralmente distinta a la nacional o autonómica, sino simplemente que hay que otorgarle el espacio y el tiempo de análisis que son necesarios (Rodríguez y Navarro, 2012).

Indagar pues, en la presencia de las mujeres en los puestos de responsabilidad política municipal puede acercarnos a entender qué características se imprimen en el ámbito local respecto al resto de niveles administrativos. Es más que patente que la infrarrepresentación de las mujeres es una problemática de extraordinaria generalidad en todos los niveles de representación política (Camas, 2013). Así pues, aunque la amplia literatura de género a este respecto pone de manifiesto que nos encontramos indudablemente ante una problemática de carácter transversal, el tratamiento del ámbito municipal parece haber quedado algo relegado, ya que no hay antecedentes de recopilación sistemática de esta información, abarcando la totalidad de las entidades locales del territorio estatal con criterios homogéneos y la finalidad explícita de analizarlos desde una perspectiva de género (FEMP, 2009: 21).

Nuestro trabajo va a basarse por tanto en el estudio de los gobiernos locales alicantinos desde una perspectiva de género, estableciendo, a su vez, una comparativa con datos de carácter 
nacional y autonómico y haciendo especial hincapié en que se han producido ya tres comicios desde que se aprobara la Ley Orgánica 3/2007, de 22 de Marzo, para la Igualdad Efectiva de Mujeres y Hombres (LOI, en adelante) en la que se recoge, entre otros, el principio de composición equilibrada en las listas electorales, introduciendo medidas correctoras, que al menos en teoría, deben favorecer la mayor presencia de las mujeres en las distintas instituciones. Así, una de las novedades más reseñables que introduce esta ley la encontramos en su disposición adicional primera, que define que:

“A los efectos de esta Ley, se entenderán por composición equilibrada la presencia de mujeres y hombres de forma que, en el conjunto a que se refiera, las personas de cada sexo no superen el sesenta por ciento ni sean menos del cuarenta por ciento", quedando de este modo establecidos mecanismos concretos para que, especialmente en el ámbito de la participación política, sea posible avanzar en la igualdad efectiva” (Soler, 2010: 87).

En cuanto a lo que a legislación valenciana se refiere, el modelo adoptado por nuestra comunidad, no establece reglas de obligatorio seguimiento en cuanto a la conformación de las listas electorales ${ }^{1}$, pero opta por favorecer la presencia de las mujeres en las candidaturas presentadas por los partidos políticos a través de diversas medidas. ${ }^{2}$

Estos cambios legislativos han traído consigo una serie de transformaciones internas en las organizaciones partidistas, que en muchos casos se han ido produciendo incluso antes de la publicación de estas leyes $^{3}$ y que han favorecido, al menos teóricamente, una mayor incorporación de las mujeres al ámbito del poder político. Con lo cual nos atrevemos a afirmar que podemos encontrarnos ante unas posibilidades de igualdad formal, pero ante una desigualdad real (Astelarra, 1990: 89).

Resultaría complicado tratar de explicar, de manera exhaustiva, la totalidad de las variables que pueden contribuir a entender las transformaciones que estamos observando al respecto del aumento de la presencia de la mujer en el ámbito político, pero sí podemos afirmar que, entre

\footnotetext{
${ }^{1}$ El artículo 10 de esta ley si establece que "Las CortsValencianes y el Consell de la Generalitat procurarán el nombramiento o designación de personas para constituir o formar parte de órganos o instituciones, para asegurar que exista una presencia paritaria de mujeres y hombres" y el art. 11 añade que "El Consell favorecerá la presencia de las mujeres en las candidaturas presentadas a las CortsValencianes por los partidos políticos y agrupaciones electorales"

${ }^{2}$ Estas serían, por ejemplo: la disposición de más tiempo gratuito en los medios de comunicación para aquellas candidaturas con presencia equilibrada de mujeres y hombres, respetando, en todo caso, las previsiones de la Ley Electoral valenciana (art. 11); las subvenciones electorales de la Generalitat reconocidas por ley se incrementarán en un $10 \%$ para los escaños obtenidos por mujeres (art. 12); y ese mismo porcentaje se aplicará en las subvenciones para cada uno de los votos conseguidos por las candidaturas con presencia equilibrada de mujeres y hombres (art. 12) (Delgado, 2011:60).

${ }^{3}$ A ello habría sin duda que sumar las normativas de los partidos políticos, que a partir de finales de los ochenta comenzarán a incluir en sus respectivos congresos medidas tendentes a la paridad, siendo las más recientes las adoptadas por IU (2012) y PSOE (2014) que abogan por listas 50\%-50\% y cremallera respectivamente.
} 
otros aspectos, parecen tener su origen en reivindicaciones de instituciones internacionales. Son numerosas las declaraciones y reivindicaciones institucionales en pro de la igualdad en la esfera política y en la toma de decisiones que se han materializado con más o menos acierto en la implementación de medidas legislativas que han jalonado la historia reciente y están vinculadas al proceso gradual de la evolución de la presencia de la mujer en la sociedad (Delgado, 2011: $32)$.

Sin embargo, es indudable que la participación de las mujeres en política es necesaria desde dos perspectivas fundamentales: por un lado, porque se trata del gobierno del pueblo, en el cual deben de estar representadas directa e indirectamente las mujeres, y por otro lado, porque su participación en la vida pública es necesaria para la eliminación de la discriminación que contra ellas se ejerce en la sociedad. La idea de democracia paritaria parte del reconocimiento del hecho de que las mujeres constituyen el 50\% de la sociedad, así, "estar en lo público" significa dos cosas: estar presente en los centros de decisión y decidir, y -algo aún más importante- ser públicas, ser vistas, ser conocidas por el público, ofrecer, en pocas palabras, una imagen de normalidad.

Y es que las mujeres constituyen, a día de hoy, más de la mitad de la población elegible, y esta proporción, no tiene en modo alguno, reflejo en la composición política de los gobiernos y de manera aún más flagrante en la esfera local, siendo, a nuestro entender, una de las áreas más importantes en cuanto a poder político se refiere, ya que se trata de una parcela sumamente operativa que puede convertirse, al tratarse del ámbito más cercano al ciudadano, en el primer paso para el avance de la participación social de las mujeres.

Aunque es cierto que desde finales de los años ochenta del pasado siglo XX hasta la actualidad, la presencia de mujeres en los puestos de responsabilidad política municipal se ha incrementado notablemente, -y de hecho, cuando hemos realizado una comparativa de los datos de las primeras elecciones locales celebradas en 1979 con los datos extraídos de las últimas, que tuvieron lugar en mayo de 2015, hemos podido constatar cómo la participación de la mujer en la política local en España se ha incrementado de una manera considerable-, sin embargo, es interesante conocer si el fenómeno es extrapolable a otros ámbitos políticos como pueden ser el nacional y el autonómico y sobre todo, poder explicar si existen diferencias significativas en ambos niveles.

A través de este estudio vamos a conocer cuál es la situación cuantitativamente hablando de las mujeres en el ámbito de decisión político más cercano a la ciudadanía, el ámbito local, que es, contrariamente a lo que se podría pensar en un principio, el más reticente a la hora de aceptar los cambios que, de manera lenta, pero al parecer progresiva, están produciéndose en todos las instituciones políticas en nuestro país.

Vamos, por tanto, a acercarnos a la realidad del poder político local en la provincia de Alicante, con un estudio de los datos referentes a la representación femenina en los gobiernos municipales de los 141 consistorios que la componen, con una delimitación temporal de treinta 
y seis años y diez convocatorias electorales locales, tres de ellas acaecidas tras la aprobación de la LOI, lo cual, pensamos, puede aportarnos datos significativos sobre los resultados de su aplicación en la esfera municipal, el ámbito más cercano y cotidiano para el/la ciudadano/a y probablemente a la vez, el menos permeable a la hora de aceptar cambios, sobre todo y a aparentemente, cuando la mayoría de municipios que lo componen son de tamaño medio-bajo, como ocurre en la provincia de Alicante, donde más del 60\% de municipios tienen menos de 5.000 habitantes y un total de 57 municipios (equivalente a casi el $40 \%$ del total) tienen menos de 1.000 vecinos/as.

\section{Presencia general de la mujer en los gobiernos locales}

Una de las categorías para el análisis de la participación de mujeres y hombres en la esfera política cuyo uso se ha empezado a generalizar es el concepto de paridad, que alude a la equidad en la representación de ambos sexos.

El concepto de paridad, suele tener un referente numérico (que se traduce generalmente en que, al menos, ningún sexo tenga una representación inferior al 40\%) pero no se agota en éste. La paridad en la representación política se enmarca en la estrategia global de promoción de la igualdad de género.

En esta estrategia global, el acceso de las mujeres a puestos de responsabilidad en la esfera política, constituye, por un lado, la traducción directa del ejercicio efectivo de uno de los derechos fundamentales de ciudadanía, y la garantía de la existencia de un sistema verdaderamente democrático; por otro lado, y desde el punto de vista de la consecución efectiva de la igualdad entre mujeres y hombres, tiene importantes implicaciones: la presencia de mujeres en puestos de toma de decisiones, contribuye a la transformación de las funciones del rol de género tradicional, generando modelos alternativos (y más igualitarios) de ser mujer que oponer a los interiorizados por la socialización diferencial, sentando así bases de futuro para la consecución de la igualdad real (FEMP, 2009: 19).

$\mathrm{Y}$ es precisamente en relación con la medición de los avances hacia la igualdad real desde donde se acuña el concepto de la brecha de género, para hacer referencia a la diferencia entre las tasas masculina y femenina en la categoría de una variable.

Las puntuaciones obtenidas nos indican el "tamaño" de esta diferencia (desigualdad): cuanto menor sea la brecha de género, más cerca estaremos de posiciones igualitarias. En este caso, cuanto menores sean las brechas de género en los espacios de poder local, más próximo estaremos del cumplimiento de este principio de "paridad". 
Tabla $n^{0} 1$. Evolución de la presencia de mujeres en la cabeza de los gobiernos locales de la Provincia de Alicante. Porcentaje y números absolutos 1979-2015

\begin{tabular}{|l|c|c|c|c|c|c|c|c|c|c|}
\hline Elecciones & $\mathbf{1 9 7 9}$ & $\mathbf{1 9 8 3}$ & $\mathbf{1 9 8 7}$ & $\mathbf{1 9 9 1}$ & $\mathbf{1 9 9 5}$ & $\mathbf{1 9 9 9}$ & $\mathbf{2 0 0 3}$ & $\mathbf{2 0 0 7}$ & $\mathbf{2 0 1 1}$ & $\mathbf{2 0 1 5}$ \\
\hline Alcaldes & 137 & 137 & 137 & 134 & 132 & 125 & 120 & 107 & 114 & 121 \\
\hline Alcaldesas & 1 & 1 & 2 & 7 & 9 & 16 & 24 & 34 & 27 & 20 \\
\hline Suma & 138 & 138 & 139 & 141 & 141 & 141 & 141 & 141 & 141 & 141 \\
\hline \% Alcaldesas & $\mathbf{0 , 7 2}$ & $\mathbf{0 , 7 2}$ & $\mathbf{1 , 4 4}$ & $\mathbf{4 , 9 6}$ & $\mathbf{6 , 2 9}$ & $\mathbf{1 1 , 1 1}$ & $\mathbf{1 6 , 6 7}$ & $\mathbf{2 3 , 6 1}$ & $\mathbf{1 8 , 7 5}$ & $\mathbf{1 4 , 1 8}$ \\
\hline \% Alcaldes & 99,28 & 99,28 & 98,56 & 95,04 & 93,71 & 88,89 & 83,33 & 76,39 & 81,25 & 85,82 \\
\hline
\end{tabular}

Fuente: Elaboración propia a partir de los datos obtenidos en la página Web de la Diputación Provincial de Alicante ${ }^{4}$

Realizando un análisis de los datos de nuestra provincia, nos encontramos con las primeras elecciones locales de nuestra reciente historia democrática, donde el porcentaje de mujeres electas para ostentar la cabeza del gobierno alicantino fue del 0,72\%. De los 138 municipios que en ese año conformaban la provincia de Alicante, tan sólo uno (concretamente, el municipio de Torrevieja) fue gobernado por una mujer en estos primeros comicios municipales.

En las siguientes elecciones, de 1983, los datos no son mucho más alentadores, manteniéndose exactamente igual el porcentaje de representación. En los terceros comicios, celebrados el 10 de Junio de 1987se produce un leve ascenso (1,44\%) y dos alcaldesas (en Calpe y Xaló). En las elecciones celebradas el 26 de Mayo de 1991, comenzamos a vislumbrar un leve cambio, ya en Alicante las féminas supondrán casi el 5\%, prácticamente triplicándose el porcentaje anterior llegando a ser siete las alcaldesas electas por esta provincia.

En los quintos comicios locales (veinte años después de las primeras elecciones municipales), se sigue constatando una trayectoria ascendente aunque claramente insuficiente. En la provincia de Alicante resultarán electas un total de nueve alcaldesas, lo que supondrá un porcentaje del 6,29\%. Dicho crecimiento comienza a constatarse de una manera más clara en las elecciones de 1999, donde ya se cuenta con un total de 16 regidoras, lo que supone poco más del $11 \%$ del total y un aumento, con respecto a las anteriores, de casi cinco puntos porcentuales. Serán estas elecciones, sin lugar a dudas, las que parecen suponer el inicio de una significativa (aunque lenta) carrera ascendente.

En cuanto a las elecciones de 2007, con la LOI ya aprobada, los datos muestran un claro ascenso, alcanzando la representación femenina casi un $24 \%$, con un total de 34 alcaldesas frente a 111 alcaldes, siendo la tasa más alta de toda la serie histórica y comenzando a descender a partir de entonces al sufrir una caída de casi cinco puntos, en las elecciones de 2011. Este cambio de tendencia queda claramente de relieve tras constatar, con los datos de los

${ }^{4}$ Unidad de Documentación [en línea] Disponible en: www.dip-alicante.es/documentacion [01/12/2015]. 
últimos comicios municipales, un descenso de más de cuatro punto con respecto a las elecciones anteriores, pasando de 27 a tan sólo 20 nuevas alcaldesas, siendo el registro más bajo desde 1999.

Si analizamos estos datos en el caso de Alicante, y una vez aprobada la LOI para el año 2011 la brecha de género y si consideramos que una brecha de género ideal debería oscilar entre 0 y 20 puntos, advertimos que en nuestra provincia la diferencia se situó en más de 62 puntos a favor de los hombres. A partir de este hecho, queda constatado que existe un clara segregación vertical hacia las mujeres, ya que, conforme tratan de avanzar en puestos de responsabilidad, más se agranda esta brecha y es difícil que no tropiecen con el ya famoso "techo de cristal". Si nos detenemos en los recientes datos de las elecciones locales de Mayo de 2015, en contra de lo que podría esperarse, en nuestra provincia esa brecha se acentuó todavía más, situándose en más de 71 puntos la sobrerrepresentación masculina al frente de los gobiernos locales.

Otro aspecto reseñable es el descubrimiento de que el 5,6\% de los municipios alicantinos (ocho en total) no tienen ningún tipo de representación política femenina en sus corporaciones locales $^{5}$, mientras, que de manera curiosa, no se da en ninguna de las localidades de la provincia la situación inversa.

\section{Gráfico 1. Evolución del acceso de la mujer a la alcaldía de las corporaciones municipales en la provincia de Alicante}

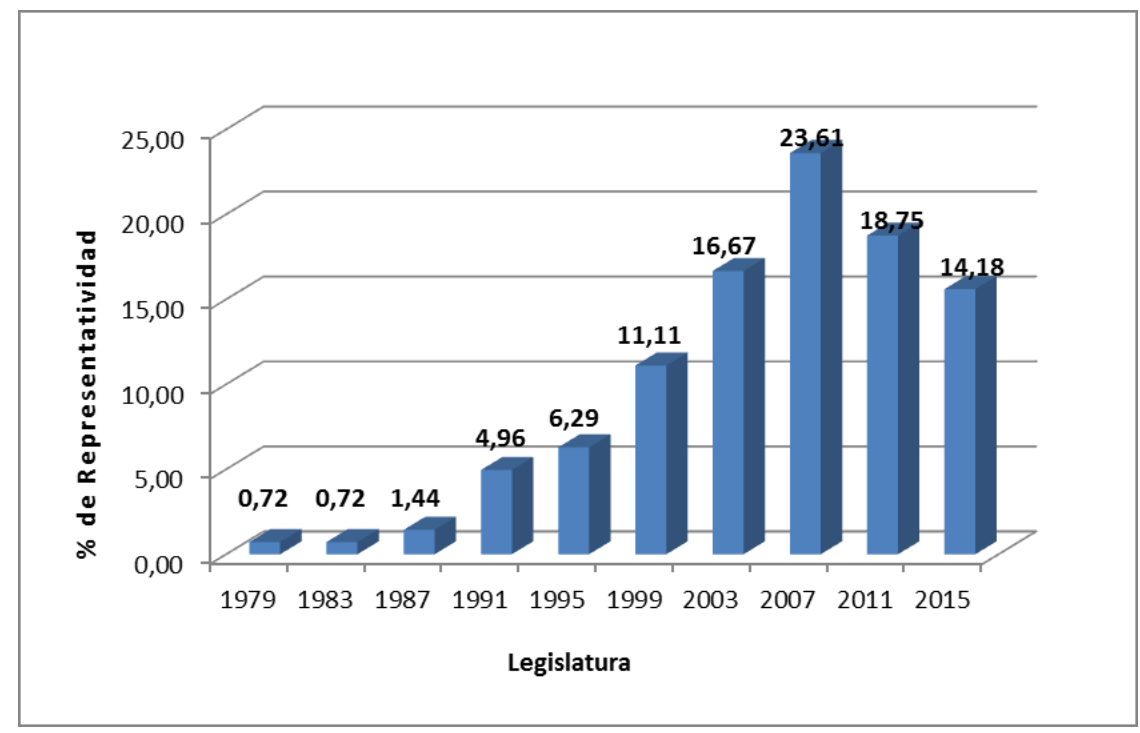

Fuente: Elaboración propia a partir de los datos recogidos en la página Web de la Diputación Provincial de Alicante 6

\footnotetext{
${ }^{5}$ Nos referimos a los municipios de Almudaina, Benillup, Confrides, Famorca, Sanet y Negrals, Tárbena, Tollos y Vall de Alcalá.

${ }^{6}$ Unidad de Documentación [en línea] Disponible en: www.dip-alicante.es/documentacion [05/08/2015].
} 
Estos datos, contrastados con los de la comunidad autónoma y los de carácter nacional quizás puedan darnos una reflexión más certera de cuál es la situación real de la provincia de Alicante.

Tabla 2. Evolución de la presencia de alcaldesas en la Comunidad Valenciana (1979-2015)

\begin{tabular}{|c|c|c|c|c|c|c|c|c|c|c|}
\hline & $\mathbf{1 9 7 9}$ & $\mathbf{1 9 8 3}$ & $\mathbf{1 9 8 7}$ & $\mathbf{1 9 9 1}$ & $\mathbf{1 9 9 5}$ & $\mathbf{1 9 9 9}$ & $\mathbf{2 0 0 3}$ & $\mathbf{2 0 0 7}$ & $\mathbf{2 0 1 1}$ & $\mathbf{2 0 1 5}$ \\
\hline Alcaldes & 541 & 536 & 522 & 564 & 553 & 524 & 520 & 446 & 438 & 427 \\
\hline Alcaldesas & 3 & 5 & 8 & 22 & 38 & 77 & 83 & 96 & 104 & 115 \\
\hline Totales & 544 & 541 & 530 & 586 & 591 & 601 & 603 & 542 & 542 & 542 \\
\hline Alcaldesas\% & $\mathbf{0 , 5 5}$ & $\mathbf{0 , 9 2}$ & $\mathbf{1 , 5 1}$ & $\mathbf{3 , 7 5}$ & $\mathbf{6 , 4 3}$ & $\mathbf{1 2 , 8 1}$ & $\mathbf{1 3 , 7 6}$ & $\mathbf{1 7 , 7 1}$ & $\mathbf{1 9 , 1 9}$ & $\mathbf{2 1 , 2 1}$ \\
\hline Alcaldes\% & 99,45 & 99,08 & 98,49 & 96,25 & 93,57 & 87,19 & 86,24 & 82,29 & 80,81 & 78,79 \\
\hline
\end{tabular}

Fuente: Elaboración propia a partir de los datos obtenidos del Ministerio de Hacienda y Administraciones Públicas ${ }^{7}$

Tabla $n^{0}$ 3. Evolución de la presencia de mujeres en la cabeza de los gobiernos locales a nivel nacional (1979-2015)

\begin{tabular}{|c|c|c|c|c|c|c|c|c|c|c|}
\hline & $\mathbf{1 9 7 9}$ & $\mathbf{1 9 8 3}$ & $\mathbf{1 9 8 7}$ & $\mathbf{1 9 9 1}$ & $\mathbf{1 9 9 5}$ & $\mathbf{1 9 9 9}$ & $\mathbf{2 0 0 3}$ & $\mathbf{2 0 0 7}$ & $\mathbf{2 0 1 1}$ & $\mathbf{2 0 1 5}$ \\
\hline Alcaldes & 8362 & 8223 & 8030 & 8302 & 8088 & 7877 & 7604 & 6880 & 6723 & 6570 \\
\hline Alcaldesas & 101 & 167 & 266 & 444 & 598 & 886 & 1142 & 1232 & 1355 & 1546 \\
\hline Totales & 8463 & 8390 & 8296 & 8746 & 8686 & 8763 & 8746 & 8112 & 8078 & 8116 \\
\hline Alcaldesas\% & $\mathbf{1 , 1 9}$ & $\mathbf{1 , 9 9}$ & $\mathbf{3 , 2 1}$ & $\mathbf{5 , 0 8}$ & $\mathbf{6 , 8 8}$ & $\mathbf{1 0 , 1 1}$ & $\mathbf{1 3 , 0 6}$ & $\mathbf{1 5 , 1 9}$ & $\mathbf{1 6 , 7 7}$ & $\mathbf{1 9 , 0 5 \%}$ \\
\hline Alcaldes\% & 98,81 & 98,01 & 96,79 & 94,92 & 93,12 & 89,89 & 86,94 & 84,81 & 83,23 & $80.95 \%$ \\
\hline
\end{tabular}

Fuente: Elaboración propia a partir de los datos obtenidos del Instituto de la Mujer y para la Igualdad de Oportunidades $^{8}$

\footnotetext{
${ }^{7}$ Secretaría de Estado de Administraciones Públicas. Unidad de Documentación [en línea] Disponible en: http://www.seap.minhap.gob.es/es/areas/politica_local.html [12/01/2016].
${ }^{8}$ Unidad
de
Documentación
[en línea]
http://www.inmujer.gob.es/estadisticas/consulta.do?area=8 [12/01/2016].
Disponible en 
Estas primeras elecciones locales, a nivel nacional nos muestran que solamente $101 \mathrm{de}$ 8.643 fueron mujeres (1,19\%). En la Comunidad Valenciana, la provincia de Castellón no contó con ninguna mujer en sus alcaldías. Además, se eligieron dos alcaldesas en Valencia y una en Alicante. La presencia total de las alcaldesas en esta comunidad fue de un 0,55\% (un total de tres mujeres por 541 hombres). La situación en nuestra provincia se colocó, por tanto, a un nivel por debajo de la media nacional, pero ligeramente superior a la media de la comunidad.

En los segundos comicios la representación femenina a nivel nacional apenas llegó al 2\%, lo que se traduce en 167 alcaldesas frente a 8.223 alcaldes (1,99\%). Sin embargo, puede considerarse un buen dato si lo comparamos con el $0,92 \%$ de la media de la comunidad o el todavía estancado $0,72 \%$ de nuestra provincia.

A partir de las elecciones de 1987 empieza a vislumbrarse un progresivo aumento de la representación femenina a nivel nacional, aunque, como en las dos legislaturas anteriores, la inmensa mayoría de las alcaldías fueron ocupadas por hombres. En esta tercera legislatura, los alcaldes representarán un 96,79\% frente al 3,21\% que representan las alcaldesas. Volvemos a situarnos en la provincia por debajo de la media nacional. A nivel autonómico, las ocho alcaldesas de la Comunidad Valenciana, representan el 1,5\% del total de las alcaldías de esta comunidad. Y en la provincia, por primera vez, nos situamos por debajo de la media tanto nacional como autonómica con tan sólo dos mujeres en la cabeza de los gobiernos locales de la provincia.

Es en los comicios de 1995 cuando los datos comienzan a igualarse y tanto en la Comunidad Autónoma como en la provincia seguirán una tendencia ascendente que, al contrario de lo sucedido con anterioridad, hará que Alicante se sitúe por encima de la media nacional en cuanto a mujeres en la cabeza de los gobiernos locales, tendencia que sólo se romperá en este último proceso electoral.

La tendencia ascendente de manera progresiva a partir de estas elecciones quizás podría explicarse porque es a finales de los ochenta, cuando los diferentes partidos políticos, en sus respectivos congresos, y siguiendo la estela de las directrices de la Unión Europea, comienzan a debatir el sistema de cuotas. Así, el PSOE introdujo en 1988 la cuota femenina del 25\%, un año más tarde le seguiría IU, y posteriormente, en 1994, el PSOE garantizaría una reserva de puestos superior en cinco puntos cuando la afiliación femenina en un territorio determinado fuera mayor del 25\% (Verge, 2006:173). Por tanto, todo parece indicar que los pocos partidos que iniciaron este camino, tuvieron el mérito de producir un efecto multiplicador al propiciar la toma de posición en el seno de otros partidos políticos que concluyeron, en muchos casos en la adopción de medidas análogas.

En este diagrama comparativo puede observarse de manera mucho más gráfica y como dato más relevantes a nuestro entender, cómo, desde 1979 hasta las elecciones de 1995, la representación femenina en los gobiernos locales a nivel nacional superará a la autonómica y a la alicantina, aunque funcionando de una manera prácticamente paralela hasta las elecciones de 1995, donde se produce un vuelco y las tendencias se invierten, pasando a destacar el aumento 
de mujeres al frente de los gobiernos locales en nuestra provincia frente a los valores autonómico y nacional. Dato que, de manera paradójica, volverá a invertirse, aunque de manera mucho más moderada en los siguientes comicios de 2011, donde se situarán de nuevo los datos nacionales por encima de los alicantinos. Tras las elecciones del 2015 el vuelco es aún mayor, ya que nos distanciamos, con respecto a la media nacional en más de cinco puntos, casi 5,5 puntos por debajo de la media nacional está la provincia de Alicante y pasando a un escasísimo $14,18 \%$.

\section{Gráfico 2. Evolución del porcentaje de ayuntamientos gobernados por mujeres a niveles Nacional, Comunidad Valenciana y Provincia de Alicante}

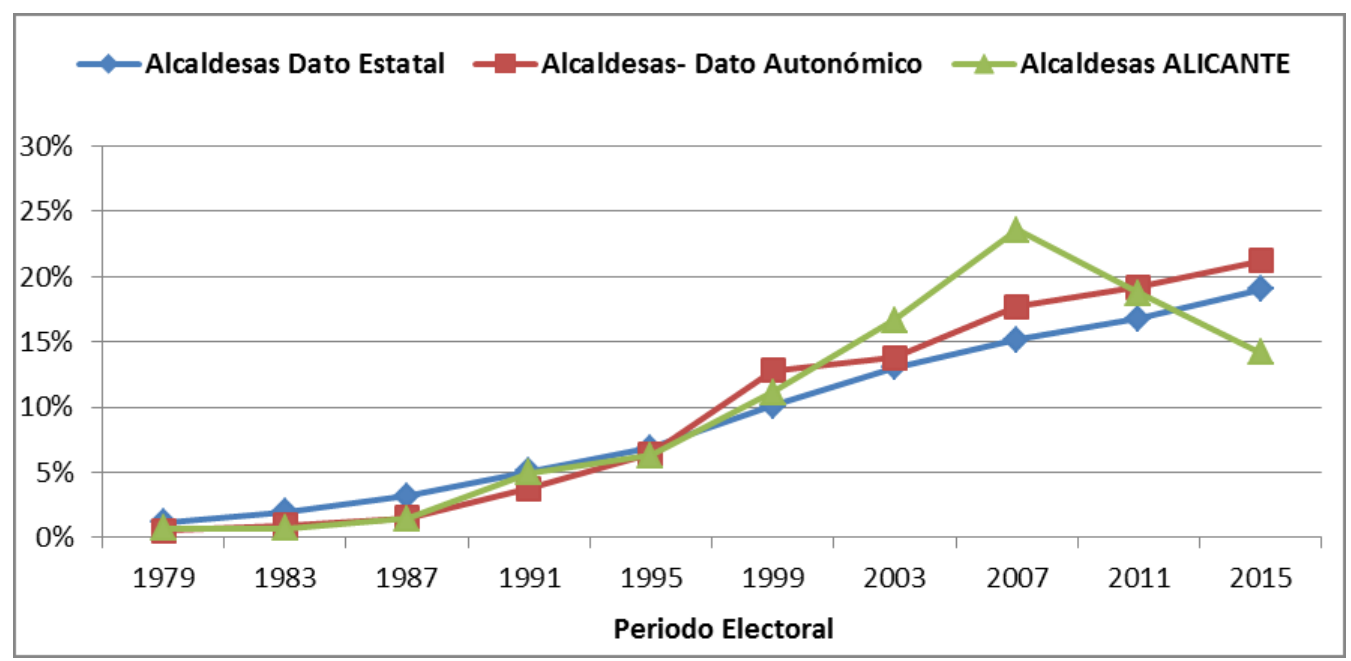

Fuente: Elaboración propia a partir de datos obtenidos de la Diputación Provincial de Alicante y del Ministerio de Hacienda y Administraciones Públicas

\section{Análisis a partir de la aprobación de la LOIMH}

La aprobación de la Ley Orgánica 3/2007, de 22 de marzo, para la Igualdad Efectiva de Mujeres y Hombres, constituye un hito sin precedentes en el establecimiento de un marco legislativo garante de una intervención institucional que promueva activamente la igualdad de género en los niveles de toma de decisiones, es por ello que consideramos que los resultados electorales producidos tras su implementación merecen una atención especial, para poder dilucidar si esta ley ha tenido impacto de manera efectiva a la hora de conseguir la tan ansiada paridad.

Sin embargo, es interesante estudiar cómo esta ley puede o no haber conseguido los resultados que teóricamente se pretendían con su implantación. Así, hay autores que señalan 
que realmente, con este sistema de cuota, no sólo no se garantiza la paridad de género del $50 \%$ de cada sexo en la representación política, sino tampoco la participación equilibrada de al menos el $40 \%$ de cada sexo:

"En efecto, la cuota de no menos del $40 \%$ de ambos sexos cada cinco puestos permite que en las candidaturas se coloquen a las mujeres en los dos últimos puestos del tramo, o bien en el último puesto cuando se trata de circunscripciones con menos de cinco puestos, [...] Así, "la fórmula del sistema de cuota electoral que adopta la LOIMH puede no asegurar la efectiva representación equilibrada de género en los órganos electos, como de hecho ha sucedido" (Saldaña,2008: 108).

Para poder contrastar estas afirmaciones, vamos a analizar los resultados de las cuatro últimas elecciones locales (2003, 2007, 2011 y 2015) en la provincia de Alicante, tratando de explicar si encontramos o no diferencias significativas entre los séptimos comicios municipales (2003) donde todavía no se había implantado el sistema de cuotas exigido por Ley y los siguientes (2007, 2011y 2015) donde ya se hallaba implantada dicha normativa.

En una primera aproximación, observamos que hay un aumento porcentual de más de siete puntos en las elecciones de 2007 con respecto a las convocatorias anteriores (es el porcentaje más elevado de diferencia entre dos periodos electorales consecutivos en todo el periodo constitucional en nuestra provincia), con lo cual, en un principio, podríamos afirmar que la ley de cuotas parece tener parte del efecto deseado, aunque aún lejano al $40 \%$ y sin llegar nunca a alcanzar el 30\% de la denominada "masa crítica" de mujeres en la élite política, a partir del cual se ha argumentado que, al menos de manera teórica, repercute positivamente a la hora de influir y adquirir protagonismo en torno a los intereses y necesidades de un grupo; en este caso las mujeres, en el momento de priorizar los asuntos y las propuestas con enfoque de género desde la institucionalidad política (Lovenduski, 1997).

No obstante, nos encontramos con la paradoja de los datos obtenidos en los comicios locales de 2011, donde, en lugar de seguir ascendiendo dicha proporción, como sería esperable, se produce un retroceso importante de representatividad femenina (se pasa del 23,61 al 18,75\%, lo que supone, en términos absolutos descender de 34 a 27 alcaldesas y una pérdida de casi cinco puntos) en esta legislatura, la cual rompe totalmente con la dinámica ascendente de los últimos treinta años. En lo que respecta al dato del 2015 para la provincia de Alicante, los resultados son muy poco alentadores: continua la tendencia descendente que ya se observó desde el 2007 y en clara contradicción con lo que sucede tanto a nivel nacional como autonómico, dónde la tendencia es ascendente.

En las elecciones municipales de 2015 nos encontramos con una bajada aún más significativa en los puestos a la cabeza de los gobiernos locales por parte de mujeres en nuestra provincia, ya que pasamos a un escasísimo 14,18\% y una representatividad de sólo 20 mujeres en toda la provincia dirigiendo nuestros consistorios frente a 121 hombres. 
Puede interpretarse que existen factores que expliquen y especifiquen las diferencias encontradas. Entre ellos sostenemos que uno decisivo se fundamentaría en la forma mediante la cual las listas de candidaturas son elaboradas, es decir, en la importancia que tienen los partidos políticos a la hora de elaborar las candidaturas electorales que concurrirán a las elecciones. En un país donde nuestro sistema electoral de tipo proporcional se decantó por la presentación de candidaturas en listas cerradas y bloqueadas, es indudable el poder que ostentan los dirigentes de los partidos políticos, que serán, en última instancia quienes van a decidir quiénes pueden tener opción a ser elegidos/as y quiénes no. Es obvio que en unas cúpulas, dirigidas mayoritaria y tradicionalmente por hombres, la mujer tendrá difícil cabida.

En definitiva, y como hemos comentado anteriormente, la LOI parece haber conseguido un impacto positivo (que no paritario) pero en absoluto determinante. En este sentido, este desequilibrio no es atribuible a la normativa, si no precisamente a que la gran mayoría de las listas electorales están encabezadas por un hombre. Mientras el ámbito de la ley en este aspecto se aplica sobre los tramos de las listas de candidaturas, será decisión del partido político la determinación final sobre quién encabeza ésta. Sólo esto podría explicar los vaivenes más que significativos que se dan en la representatividad al cargo de los consistorios en esta provincia y el claro descenso de alcaldesas una vez aprobada la LOI.

Para finalizar este análisis, creemos que es importante estudiar los datos atendiendo a los diferentes partidos políticos a los que pertenecen las regidoras que gobiernan en la provincia, para ver si se cumple la máxima de si, los partidos de izquierdas, primeros en implementar las cuotas y en apostar por valores de equidad, sitúan realmente a las mujeres en los puestos de salida.

\section{Grafico 3. Porcentaje de alcaldesas según partido político en la Provincia de Alicante 2003-2015}

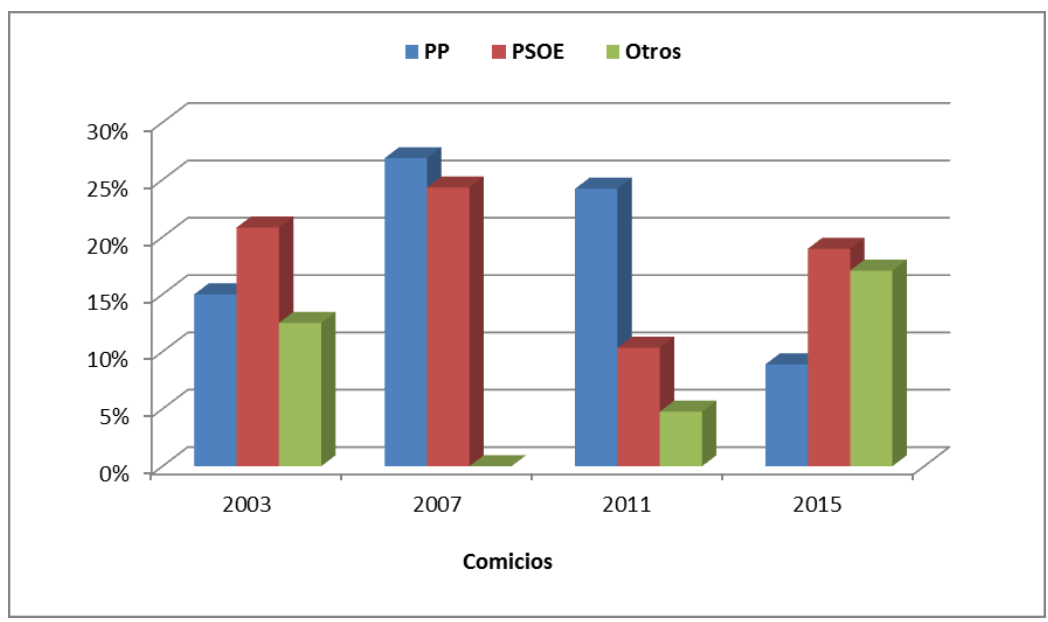

Fuente: Elaboración propia a partir de datos obtenidos de la Diputación Provincial de Alicante ${ }^{9}$

${ }^{9}$ Unidad de Documentación [en línea] Disponible en www.dip-alicante.es/documentacion [01/02/2016] 
Tabla 4. Alcaldesas por partido político en la provincia de Alicante (2003-2015)

\begin{tabular}{|c|c|c|c|c|c|c|c|c|c|c|c|c|}
\hline & \multicolumn{3}{|c|}{ Año 2003 } & \multicolumn{3}{c|}{ Año 2007 } & \multicolumn{3}{c|}{ Año 2011 } & \multicolumn{3}{c|}{ Año 2015 } \\
\hline & PP & PSOE & Otros & PP & PSOE & Otros & PP & PSOE & Otros & PP & PSOE & Otros \\
\hline Alcaldesas & 12 & 10 & 2 & 25 & 9 & 0 & 23 & 3 & 1 & 4 & 9 & $7^{10}$ \\
\hline Alcaldes & 68 & 38 & 14 & 68 & 28 & 14 & 72 & 26 & 20 & 41 & 49 & $31^{11}$ \\
\hline Totales & 80 & 48 & 16 & 93 & 37 & 14 & 95 & 29 & 21 & 49 & 66 & 44 \\
\hline \% Mujeres & 15,00 & 20,83 & 12,50 & 26,88 & 24,32 & 0,0 & 24,21 & 10,34 & 4,76 & 8,16 & 13,33 & 13,63 \\
\hline \% Hombres & 85,00 & 79,17 & 87,50 & 73,12 & 75,68 & 100 & 75,79 & 89,66 & 95,24 & 91,84 & 86,67 & 86,37 \\
\hline
\end{tabular}

Fuente: Elaboración propia a partir de datos obtenidos de la Diputación Provincial de Alicante ${ }^{12}$

El mayor porcentaje de alcaldesas electas pertenecen, en los comicios previos a la aprobación de la LOI al PSOE, que ya había asumido en su XXXIV Congreso las políticas de cuotas, lo que sumado al porcentaje de mujeres regidoras en el apartado "Otros" que comprende en las tres legislaturas, y de manera absolutamente mayoritaria, a partidos de izquierda nacionalista valencianos ${ }^{13}$, nos da un total de más del $33 \%$ frente al $15 \%$ de alcaldesas del PP. Queda por tanto demostrado a través de los datos que también en nuestra provincia, son los partidos de sesgo ideológico más progresista los que obtiene para las mujeres mayores índices de representatividad, al menos hasta la obligatoriedad de la ley.

Será a partir de la implementación de cuotas por la LOI cuando los datos se inviertan y el PP presentará mayor porcentaje de regidoras electas $(26,88 \%)$ con un ascenso de más de once puntos porcentuales con respecto a las elecciones anteriores, mientras que en el PSOE la subida es ligeramente inferior, pasando del 20,83 a poco más del $24 \%$ en los comicios de 2007.

Pero sin duda, son los datos de las elecciones de 2011 los que vuelven a llamarnos la atención, ya que, en términos generales, el número de mujeres alcaldesas desciende en los dos partidos mayoritarios de manera ostensible, con descenso moderado del PP y más de 14 puntos del PSOE. Una de las posibles explicaciones a este fenómeno podría basarse en las

\footnotetext{
${ }^{10}$ Señalar la importancia de Compromís, que irrumpe en la provincia con 3 alcaldesas de esas 7, a sólo una de distancia del Partido Popular. Las otras 4pertenecen: 1 a Esquerra Unida del País Valencià y las otras 3 a formaciones independientes.

${ }^{11} 16$ de ellos de Compromís. Las alcaldesas de Compromís en la provincia (3) supondrían un 15,78\% frente a los alcaldes $(84,22 \%)$, el porcentaje más alto de entre todos los partidos si lo estudiáramos de manera individual.

${ }^{12}$ Unidad de Documentación [en línea] Disponible en www.dip-alicante.es/documentacion [01/02/2016]

${ }^{13}$ Bloc Nacionalista Valencià (2003 y 2007), y Coalició Compromís $(2011,2015)$.
} 
previsiones electorales, es probable que el Partido Socialista Obrero Español, ante la debacle electoral que le auguraban las encuestas para la provincia de Alicante, decidiera destinar los pocos puestos "seguros" a hombres, o visto desde otro punto de vista, cabe la posibilidad de que consideraran que el situar a un hombre como cabeza de lista podría darles más posibilidades de éxito electoral.

En cuanto al apartado "Otros" se recupera levemente pero en ningún caso vuelve a alcanzar los porcentajes de las elecciones de 2003, aunque tendrá un enorme repunte tras los comicios de 2015.

En vista de estos datos, podría afirmarse que la LOI sí ha influido de manera notable en un aumento de mujeres al frente de los consistorios en nuestra provincia en las elecciones de 2007 sobre todo en el PP. Consideramos que el incremento tan importante de alcaldesas que se produce en el Partido Popular puede deberse a que, por primera vez, se ven obligados/as a acatar por ley unas cuotas que per se, no entran ni en su ideario ni mucho menos en sus estatutos, con lo que los porcentajes de partida son mucho más bajos que los de sus homólogos progresistas que ya tenían implantados de una manera u otra políticas de cuotas en sus respectivas formaciones.

En lo que respecta a los últimos comicios municipales dos aspectos reseñables a nuestro entender: por un lado el enorme descenso de alcaldesas populares, en más de 16 puntos pasando del $24,2 \%$ al $8,1 \%$, de 23 a 4 alcaldesas, un ligero ascenso del PSOE, que pasa de 3 a 9 (de un 10 a un 13\%) y sobre todo de "Otros", de un 4,7\% a un $13,6 \%$ en ascenso de representación femenina. Señalar la importancia de Compromís, que irrumpe en la provincia con 3 alcaldesas de esas 6, a sólo una de distancia del Partido Popular.

Consideramos que para lograr una mayor presencia en puestos relevantes en las listas que impliquen la posibilidad de acceso a cuotas de poder, es necesario que en el espacio político coexistan rutinas sociales que acrecienten y prescriban cambios culturales, ya sean referidos a concepciones enmarcadas en estereotipos y prejuicios sexistas o a la influencia de las estructuras institucionales y la cultura política en las oportunidades femeninas para acceder al poder. El partido político es el responsable del reclutamiento y la selección de las candidatas y los candidatos, y la superación de estas barreras pasa por la interiorización por parte de las ejecutivas de la selección de éstos/as de forma democrática, justa y eficiente, ya que es por todos/as asumido que el aumento progresivo de la participación de la mujer en puestos de toma de decisión puede influir positivamente en la dirección de ese necesario cambio cultural en curso, de tal forma que la existencia de voces femeninas en el seno de los partidos políticos y en el sistema representativo en general, tal y como señalan Diz y Lois (2004: 218), tiende a romper con la indiferencia acerca del papel de las mujeres en la vida pública. 


\section{Conclusiones}

En este artículo hemos presentado un estudio de los gobiernos locales de la provincia de Alicante desde una perspectiva de género, analizando la presencia de mujeres en las alcaldías, a través de la comparación de datos provinciales, autonómicos y estatales.

Tal y como interpretan acertadamente Uriarte y Ruiz (1999: 221), únicamente los análisis de listas y resultados electorales no aportan información suficiente para conocer la raíz del problema de la subrepresentación femenina. Y aunque consideramos que la relevancia de este tipo de trabajos está limitada a la cuantificación y descripción de este proceso social a través de sus resultados tangibles, por lo tanto a la posición y cantidad de mujeres en una lista electoral, creemos sin embargo que es un indicador más que fiable del grado de integración de la mujer en la sociedad. Sólo por esto, este tipo de estudios ya está justificado. Si bien no se pueden derivar explicaciones de por qué se llega a la discriminación hallada en los datos, sí que se indica dónde hay que buscar las causas: en las prácticas de reclutamiento de los partidos políticos y en los factores ambientales que las condicionan.

Tras el análisis de datos realizado, podemos afirmar que de manera general se han conseguido importantes avances en términos de igualdad tanto a nivel nacional como en la provincia de Alicante, superando incluso, a partir del año 1995 a los datos estatales. Sin embargo, la caída aunque en principio sea moderada a partir de los dos últimos comicios locales y claramente acentuada tras las últimas elecciones municipales, nos alejan bastante de la media nacional $^{14}$ y nos hace por tanto plantearnos cuál puede ser la efectividad a más largo plazo de la implementación de cuotas, ya que de nuestra investigación se deduce que, aunque sobre el papel se está cumpliendo la legislación electoral, no se sitúa un número suficiente de mujeres en las cabezas de lista de los partidos políticos, ocupando éstas de manera mayoritaria los puestos posteriores, ya que en ningún momento se consigue alcanzar esa proporcionalidad, y en muchos casos ni siquiera acercarnos al 30\% de masa crítica.

Por lo tanto, con los resultados que ofrece este estudio, donde en ningún proceso electoral (incluidos los posteriores a la aprobación de la LOI) se llega a alcanzar ni siquiera el $25 \%$ de representación femenina en la cabeza de los gobiernos locales, y teniendo en cuenta los desesperanzadores datos de la provincia alicantina, que continúa, de manera imparable, esa tendencia al descenso que ya se inició desde 2011, no puede sino defenderse como posible alternativa, la cuota del $50 \%$ en tramos de dos puestos, es decir, la llamada "lista cremallera", que ya ha sido aplicada en varios regímenes electorales autonómicos con bastante buenos resultados y que la misma LOI reconoce y ampara. Y de hecho, para garantizar la validez de las leyes de paridad autonómicas vigentes, el artículo 44 bis que regula esta normativa, incorpora una cláusula que permite a las leyes autonómicas reguladoras de sus respectivos sistemas

\footnotetext{
${ }^{14}$ Recordemos que los datos son: 19,5\% alcaldesas a nivel nacional, 21, 21\% Comunidad Valenciana y un exiguo $14,18 \%$ en la provincia de Alicante.
} 
electorales la adopción de medidas que favorezcan una mayor presencia de mujeres en las candidaturas que se presenten a las elecciones de miembros de las Asambleas Legislativas de las Comunidades Autónomas ${ }^{15}$.

Estamos convencidas, de que para asegurar la efectiva transversalidad de la presencia equilibrada de género en la toma de decisiones a todos los niveles, será necesario definir y adoptar estrategias concertadas que impliquen no sólo al Estado, (demostrándose que la legislación, por si sola, no produce todos los efectos deseados) sino a una amplia variedad de actores de los ámbitos político y público, económico y social. Un claro ejemplo de esta necesidad lo encontramos en el Congreso de los Diputados, que en nuestro país se estrenó con un porcentaje del $6 \%$ de mujeres en las Cortes Constituyentes, que descendería al 4,8\% en las legislativas de 1979, hasta llegar en 2011 al 35,43\%, y nos hallamos, en los últimos comicios generales con que 138 mujeres del total de 350 diputados que han sido elegidos son mujeres. Este $39,4 \%$ es la cifra más alta desde 1979 y la más cercana a la equidad desde que se inició el actual periodo democrático ${ }^{16}$.

Entendemos pues, que por una parte son los propios partidos políticos los que deben cambiar sus estrategias de reclutamiento y conformación de listas electorales, y de hecho los datos nos han demostrado que, en las formaciones políticas que se realizan primarias o tienen establecido algún tipo de sistema de obligado cumplimiento en cuanto a cuotas, los resultados son muchísimo más cercanos a la paridad. Además creemos interesante resaltar los efectos positivos en cuanto a la igualdad se refiere de la irrupción de los nuevos partidos, en especial de Podemos ${ }^{17}$, que son los que presentan, mejores datos en cuanto a diputadas electas a nivel nacional y a Compromís, que es el que muestra a nivel provincial mayores tasas de alcaldesas de entre todas las formaciones políticas que concurrieron a los comicios locales ${ }^{18}$.

Por otra parte, consideramos que entre las distintas actuaciones encaminadas a conseguir dicho objetivo de acercamiento a la paridad en los puestos de decisión, entendemos que es necesario el fomento de la investigación sobre la participación política de las mujeres, ahondar en las barreras que impiden esa participación, y, sobre todo, buscar e implementarlas estrategias

\footnotetext{
${ }^{15}$ Así nos encontramos la Ley 11/2002, de 27 de junio, de modificación de la Ley 5/1986, de 23 de diciembre, electoral de Castilla- La Mancha; la Ley 6/2002, de 21 de junio, de modificación de la Ley 8/1986, de 26 de noviembre, electoral de la Comunidad autónoma de las Islas Baleares; y la Ley 5/2005, de 8 de abril, por la que se modifica la Ley 1/1986, de 2 de enero, Electoral de Andalucía. La Ley 5/1990, de Elecciones al Parlamento Vasco, en virtud de la modificación introducida por la Ley 4/2005, de 18 de febrero, del País Vasco para la igualdad de mujeres y hombres, no establece realmente un sistema cremallera sino un cupo del $50 \%$ de mujeres por cada tramo de seis puestos en las candidaturas presentadas en las elecciones al Parlamento Vasco.

${ }^{16}$ Véanse los siguientes artículos periodísticos: a) Borraz y Sánchez (2015): "El Congreso con más mujeres diputadas de la Democracia”."El periódico.es, y b) Europapress (2015): "138 mujeres en el Congreso, la cifra más alta de la democracia"

${ }^{17}$ Recordemos que Podemos tiene un $49,28 \%$ de diputadas en el parlamento.

${ }^{18}$ Compromís presenta la tasa más alta de alcaldesas con respecto a alcaldes de todas las formaciones políticas que obtuvieron representación en los comicios locales en Alicante $(15,78 \%$, frente al 13,33\% del PSOE o el 8,16\% del PP).Cabe recordar que la marca Podemos como tal no se presentó a los comicios locales de mayo de 2015.
} 
para superarlas. El único interrogante que deberíamos plantearnos en estos momentos de incertidumbre económica y política es, hasta qué punto, los gobiernos van a estar dispuestos a invertir en estos aspectos que parecen considerar "no prioritarios".

Finalmente, la conclusión debe ser de cautela. Pensamos que los gobiernos municipales pueden constituir, en este aspecto, un campo de pruebas más que reseñable tanto para el estudio como para la aplicación de cualquier tipo de política tendente a la equidad que queramos llevar a cabo, ya que, aunque conciertas particularidades, la política local reproduce de manera bastante análoga el funcionamiento y las relaciones de poder tanto a nivel autonómico como nacional, teniendo más facilidades a la hora de su implementación como campo de actuación inicial. Además, y tal y como hemos señalado anteriormente y en contra de lo que mayoritariamente se cree, es el ámbito municipal el menos permeable a la participación femenina y los datos así lo evidencian $\left(39,4 \%\right.$ de diputadas, $41,6 \%$ de senadoras ${ }^{19}$ y $28,5 \%$ de ministras ${ }^{20}$, frente al $19,05 \%$ de alcaldesas), con lo que entendemos que debería ser, sin duda, el ámbito prioritario de actuación de los poderes públicos.

\section{BIBLIOGRAFÍA}

- Astelarra, Judith (1990) (comp.): Participación politica de las mujeres. Madrid: Siglo XXI de España Editores: Centro de Investigaciones Sociológicas (CIS).

- Borraz, Marta y Sánchez, Raúl (2015): "El Congreso con más mujeres diputadas de la Democracia". En El Periódico, 21 de diciembre, [en línea] Disponible en: http://www.eldiario.es/politica/nuevo-Congreso-contara-mujeres-diputadas_0_465103579.html [03/01/2016].

- Camas, Francisco (2013): "La presencia de las mujeres en los ayuntamientos andaluces (1979-2011)". En Colección Actualidad, nº 69, Centro de Estudios Andaluces, pp. 5-24.

- Delgado, Irene (2011) (ed.): Alcanzando el equilibrio. El acceso y la presencia de las mujeres en los parlamentos. Valencia: Tirant lo Blanch.

- Diz, Isabel y Lois, Marta (2004): "La presencia política de las mujeres. Una comparación de la clase política y la opinión pública gallegas”. En: Zona Abierta, nº. 106-107, pp. 175-223.

- Europapress (2015): “138 mujeres en el Congreso, la cifra más alta de la democracia”, 21 diciembre, [en línea] Disponible en http://www.europapress.es/epsocial/igualdad-00328/noticia20d-undecima-legislatura-arrancara-138-mujeres-congreso-cifra-mas-alta-democracia20151221142429.html [03/01/2016].

\footnotetext{
${ }^{19}$ En el que influyen sin duda, el "estreno" de listas cremallera por las que han optado PSOE, Podemos y Unidad Popular-Iu.

${ }^{20}$ Hay que señalar que a fecha actual aún nos encontramos con un gobierno en funciones, pues no se ha constituido aún nuevo gobierno tras las pasadas elecciones del 20 de diciembre de 2015.
} 
- Federación Española de Municipios y Provincias (FEMP) (2009): Impacto de la Ley Orgánica 3/2007 para la Igualdad Efectiva de Mujeres y Hombres en la Representación de Mujeres en el Ámbito de la Política Local. Madrid: FEMP.

- Lovenduski, Joni (1997): "Representación política: dinámica de géneros y partidos". En: Edurne Uriarte y Arantxa Elizondo (coords.): Mujeres en política. Análisis y práctica. Barcelona: Ariel, pp. 114-132.

- Rodríguez, Ma Jesús y Navarro, Clemente J. (2012): "La feminización de la dinámica política municipal. El caso de los municipios españoles". En: Revista Internacional de Sociología, Vol. 1, no. 70, pp. 181-201.

- Ruiloba, Juana (2013): Género y estilos de liderazgo en el gobierno local. Las alcaldesas en España. Madrid: Congreso de los Diputados.

- Salazar, Octavio (2010): "La autonomía local en serio. La bella durmiente...30 años después". En Francisco J. García y Enoch Albertí: Treinta años de Constitución. Valencia: Tirant lo Blanch, pp. 191-224.

- Saldaña, Ma Nieves (2008): “Transversalidad de la participación equilibrada de género en el marco internacional: su recepción en la ley de igualdad española". En: Feminismos, no .12 , pp. 79-134.

- Soler, Margarita (2010): "La Ley Orgánica para la igualdad efectiva de mujeres y hombres. Las leyes autonómicas de igualdad entre mujeres y hombres". En: Julia Sevilla et al:: Políticas públicas de igualdad. Castellón: Fundación Isonomía para la Igualdad de Oportunidades y Universitat Jaume I, pp. 78-117.

- Uriarte, Edurne y Ruiz, Cristina (1999): "Mujeres y Hombres en las élites políticas españolas: ¿diferencias o similitudes?”. En: REIS, n⿳0.88/99, pp.207-233.

- Verge, Tania (2006): “Mujer y partidos políticos en España: Las estrategias de los partidos políticos y su impacto institucional”. En: Revista Española de investigaciones Sociológicas, $\mathrm{n}^{\mathrm{o}}$. 115 , pp. 165-196. 